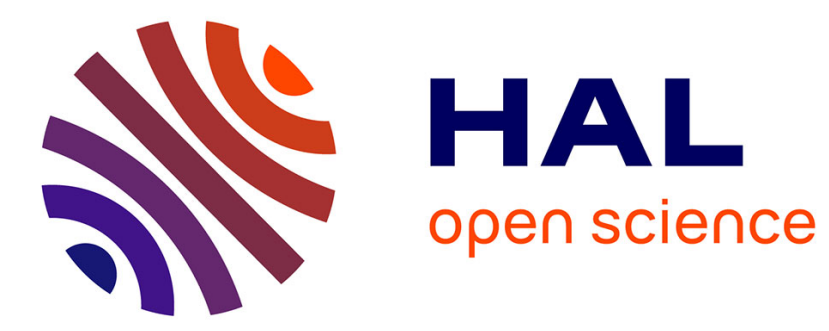

\title{
The core of bicapacities and bipolar games
}

Lijue Xie, Michel Grabisch

\section{To cite this version:}

Lijue Xie, Michel Grabisch. The core of bicapacities and bipolar games. Fuzzy Sets and Systems, 2007, 158 (9), pp.1000-1012. 10.1016/j.fss.2006.12.007 . halshs-00187162

\section{HAL Id: halshs-00187162 https://shs.hal.science/halshs-00187162}

Submitted on 13 Nov 2007

HAL is a multi-disciplinary open access archive for the deposit and dissemination of scientific research documents, whether they are published or not. The documents may come from teaching and research institutions in France or abroad, or from public or private research centers.
L'archive ouverte pluridisciplinaire HAL, est destinée au dépôt et à la diffusion de documents scientifiques de niveau recherche, publiés ou non, émanant des établissements d'enseignement et de recherche français ou étrangers, des laboratoires publics ou privés. 


\title{
The core of bicapacities and bipolar games
}

\author{
Lijue XIE and Michel GRABISCH \\ University of Paris I - Panthéon-Sorbonne \\ CERMSEM, 106-112 Bd de l'Hôpital 75103 Paris, France \\ Email: xielijue@yahoo.fr, michel.grabisch@lip6.fr
}

December 21, 2005

\begin{abstract}
Bicooperative games generalize classical cooperative games in the sense that a player is allowed to play in favor or against some aim, besides non participation. Bicapacities are monotonic bicooperative games, they are useful in decision making where underlying scales are of bipolar nature, i.e., they distinguish between good/satisfactory values and bad/unsatisfactory ones. We propose here a more general framework to represent such situations, called bipolar game. We study the problem of finding the core of such games, i.e., the set of additive dominating games.
\end{abstract}

Keywords: fuzzy measure, bicapacity, cooperative game, bipolar scale, core.

\section{Introduction}

Capacities (otherwise called fuzzy measures) and cooperative games are mathematically close concepts, although applied and studied in separate fields. Despite this fact, a similar generalization has appeared in both fields, called bicapacities on the one side [6], and bicooperative games [1] on the other side. The aim is to describe situations where some neutral level exist, surrounded by levels which are considered as bad, unsatisfactory or harmful on one side, and good, satisfactory or benefic on the other side. As for example, a player in a cooperative game can cooperate with other players to reach a given common goal (benefic action), not participate (neutral position), or do some action against the common goal (harmful action).

The first aim of the paper is to propose a general framework for this, called bipolar games, taking into account recent generalizations of games and capacities, like multichoice games, where each player can have different participation level, between full participation and non participation. This framework is presented in Section 2.

A question of importance in game theory and decision making where capacities are applied, is to find the set of additive capacities or games dominating a given capacity or game. They represent, depending on the context, the set of rational imputations for players, or the set of compatible probability measures. This set is called the core, and it 
may be empty. Necessary and sufficient conditions for nonemptiness are known, as well as the structure of the core for convex games or capacities. A recent study by the authors has been done for multichoice games, which is presented in Section 3. The second aim of the paper is to investigate about the core of bipolar games. This is described in Section 4, the main section of the paper. A last section applies the general results to the case of bicooperative games and bicapacities.

\section{Bipolar games and capacities}

Classical cooperative games on a finite set $N$ of $n$ players model situation where players form coalitions $S \subseteq N$, and to each coalition $S$ is attached a worth $v(S)$ representing the amount of money the coalition will get by itself (see, e.g. Owen [14]). Formally, a game in characteristic function form or game for simplicity is a function $v: 2^{N} \rightarrow \mathbb{R}$ such that $v(\emptyset)=0$.

The game is monotonic if whenever $S \subseteq T$, we have $v(S) \leq v(T)$. A monotonic game such that $v(N)=1$ is called a capacity [2] or fuzzy measure [15].

In a classical game, a player can either participate to some coalition or not participate. Multichoice games model situation where each player $i \in N$ can have several totally ordered levels of participation, labelled 0 (no participation), $1,2, \ldots l_{i}$ (full participation). Coalitions are then replaced by participation profiles, indicating for each player his level of participation [12]. Formally, we consider the product lattice $L:=L_{1} \times \cdots \times L_{n}$, where $L_{i}:=\left\{0,1,2, \ldots, l_{i}\right\}$ is the linear lattice of participation levels, with the obvious ordering. Then a multichoice game is a function $v: L \rightarrow \mathbb{R}$, such that $v(0,0, \ldots, 0)=0$. Multichoice games are particular cases of games on lattices, as presented in [5, 10], where each player has at disposal a set of actions which is partially ordered.

A multichoice game is monotonic if $x \leq x^{\prime}$ implies $v(x) \leq v\left(x^{\prime}\right)$, where $x, x^{\prime}$ are participation profiles in $L$, and $\leq$ is the coordinatewise order. Monotonic multichoice games with $l_{1}=l_{2}=\ldots, l_{n}=k$ are called $k$-ary capacities [7]. A multichoice game $v$ is additive if for any $x \in L, v(x)=\sum_{i \in N} v\left(0_{1}, \ldots, 0_{i-1}, x_{i}, 0_{i+1}, \ldots, 0_{n}\right)$.

Ternary voting games of Felsenthal and Machover [4] and bicooperative games of Bilbao $[1,13]$ present yet a different situation. In ternary voting games, voters can vote in favor, against or abstain. While abstention is clearly a non participation, voting in favor or against are forms of participation, leading to opposite consequences. Bicooperative games are in a sense a generalization of ternary games, which consider that players can play towards or against a given goal, or not participate. Those playing to reach the goal are called defenders, while those playing against are called defeaters. Hence we deal with bicoalitions $(S, T)$, where $S$ is the defender coalition and $T$ the defeater coalition. Introducing the notation $\mathcal{Q}(N):=\left\{(S, T) \in 2^{N} \times 2^{N} \mid S \cap T=\emptyset\right\}$, bicooperative games are functions $v: \mathcal{Q}(N) \rightarrow \mathbb{R}$ such that $v(\emptyset, \emptyset)=0$.

A bicooperative game is monotonic if whenever $S \subseteq S^{\prime}$ or $T \supseteq T^{\prime}$, we have $v(S, T) \leq$ $v\left(S^{\prime}, T^{\prime}\right)$. Monotonic bicooperative games satisfying $v(N, \emptyset)=1$ and $v(\emptyset, N)=-1$ are called bicapacities [8,9]. A specific section is devoted to bicooperative games and bicapacities (Sec. 5).

We may propose a more general framework for bicooperative games, which we call bipolar games. First we recall a few notions about lattices and ordered sets (see, e.g., [3]). 
A set $L$ endowed with a reflexive, antisymmetric and transitive relation $\leq$ is a lattice if for every $x, y \in L$, a unique least upper bound (denoted $x \vee y$ ) and a unique greatest lower bound $x \wedge y$ exist. The top element $\top$ (resp. bottom element $\perp$ ) of $L$ is the greatest (resp. the least) element of $L$, and always exist when the lattice is finite. The lattice is said to be linear or linearly ordered or totally ordered if $\leq$ is a total order on $L$. For any element $x \in L$, the principal ideal of $x$ is defined by $\downarrow x:=\{y \in L \mid y \leq x\}$, and the principal filter of $x$ is $\uparrow x:=\{y \in L \mid y \geq x\}$.

A bipolar lattice $(L, \leq)$ is a lattice with top and bottom element $\top, \perp$, such that it exists a particular element 0 called the central element, with the property that $L=\uparrow 0 \cup \downarrow 0$, i.e., each element of $L$ is either above or below the central element. We denote for convenience $L^{+}:=\uparrow 0=\{x \in L \mid x \geq 0\}$, and $L^{-}:=\downarrow 0=\{x \in L \mid x \leq 0\}$.

Let us consider $\left(L_{1}, \leq_{1}\right), \ldots,\left(L_{n}, \leq_{n}\right)$ bipolar lattices with central points $0_{1}, \ldots, 0_{n}$, top and bottom elements $\top_{1}, \ldots, \top_{n}$ and $\perp_{1}, \ldots, \perp_{n}$, and the lattice $L:=L_{1} \times \cdots \times L_{n}$, endowed with the product order, i.e, $x \leq y$ if $x_{i} \leq_{i} y_{i}$, for all $i \in N$, so that we have for infimum and supremum:

$$
\begin{aligned}
& x \vee y:=\left(x_{1} \vee y_{1}, \ldots, x_{n} \vee y_{n}\right) \\
& x \wedge y:=\left(x_{1} \wedge y_{1}, \ldots, x_{n} \wedge y_{n}\right) .
\end{aligned}
$$

Let us consider $\mathbb{O}:=\left(0_{1}, \ldots, 0_{n}\right)$, and top and bottom of $L$ which are $\top:=\left(\top_{1}, \ldots, \top_{n}\right)$ and $\perp:=\left(\perp_{1}, \ldots, \perp_{n}\right)$. Note that $(L, \leq)$ is no more a bipolar lattice since, e.g., $\left(\perp_{1}, \top_{2}, \top_{3}, \ldots, \top_{n}\right)$ is not comparable with $\mathbb{O}$. Nevertheless, we still consider $L^{+}$and $L^{-}$:

$$
\begin{aligned}
& L^{+}:=\uparrow \mathbb{O}=L_{1}^{+} \times \cdots \times L_{n}^{+} \\
& L^{-}:=\downarrow \mathbb{O}=L_{1}^{-} \times \cdots \times L_{n}^{-} .
\end{aligned}
$$

For any $x \in L, x^{+}:=x \vee \mathbb{O}$ and $x^{-}=x \wedge \mathbb{O}$ denote the positive and negative parts of $x$, elements of $L^{+}, L^{-}$respectively.

Definition 1 Let $L$ be a product of bipolar lattices as above. A bipolar game is a function $v: L \rightarrow \mathbb{R}$, with $v(\mathbb{O})=0$.

As for games on lattices, each $L_{i}$ represents the set of possible actions of player $i$. Players choosing an action in the $L_{i}^{+}$part of $L_{i}$ (i.e., above $0_{i}$ ) are defenders, those choosing an action in the $L_{i}^{-}$part are defeaters, while $0_{i}$ indicates no participation. We denote by $\mathcal{G}(L)$ the set of bipolar games on $L$.

In the sequel, we restrict to the case of linear bipolar lattices, that is, those of the form $L_{i}:=\left\{-s_{i}, \ldots,-1,0,1, \ldots, t_{i}\right\}$, with a total order $-s_{i}<\cdots<-1<0<1<\cdots<t_{i}$. Clearly, $L_{i}^{+}=\left\{0,1, \ldots, t_{i}\right\}$, and $L_{i}^{-}=\left\{-s_{i}, \ldots,-1,0\right\}$. We have $\top=\left(t_{1}, \ldots, t_{n}\right)$, $\perp=\left(-s_{1}, \ldots,-s_{n}\right)$. For convenience, we introduce the dual lattice of $L^{-}$, i.e. the set $-L^{-}:=\left\{-x \mid x \in L^{-}\right\}$endowed with the reverse order. Hence, its top and bottom elements are $\left(s_{1}, \ldots, s_{n}\right)=-\perp$ and $\mathbb{O}$ respectively. To any element $x \in L^{-}$, we make correspond $x^{\prime} \in-L^{-}$by $x_{i}^{\prime}:=x_{i}+s_{i}$ for all $i \in N$, in short:

$$
x^{\prime}:=x-\perp, \quad \forall x \leq \mathbb{O} .
$$

Hence, $\perp^{\prime}=\mathbb{O}$ and $\mathbb{O}^{\prime}=-\perp$. To any bipolar game $v$ on $L$, we associate the multichoice game $v^{-}$on $-L^{-}$by

$$
v^{-}\left(x^{\prime}\right):=v(x)-v(\perp), \quad \forall x \leq \mathbb{O} \text {. }
$$


Similarly, the multichoice game $v^{+}$is the restriction of $v$ to $L^{+}$:

$$
v^{+}(x):=v(x), \quad \forall x \geq \mathbb{O} .
$$

We may sometimes use the notation $v=\left(v^{+}, v^{-}\right)$to enhance the fact that $v$ is a bipolar game.

The particular case where $s_{i}=t_{i}=l$ for all $i \in N$ is called a l-bipolar game. Clearly, ternary voting games and bicooperative games are 1-bipolar games, with $L_{1}=L_{2}=\cdots=$ $L_{n}=\{-1,0,1\}$.

To simplify the notations, we denote the element where the $i$-th player plays at the $k$-th action level, the others doing nothing, by $\left(k_{i}, 0_{-i}\right)$ or even $\widetilde{k_{i}}$. More generally, we use the notation $\left(k_{i}, x_{-i}\right)$, where $x_{i} \in L_{-i}:=\prod_{j \neq i} L_{j}$.

Remark that for any $i \in N$ and $x_{i}, y_{i} \geq 0,\left(-s_{i}+x_{i}\right) \vee\left(-s_{i}+y_{i}\right)=-s_{i}+\left(x_{i} \vee y_{i}\right)$, and the same holds with $\wedge$ replacing $\vee$. Hence we get, for any $x, y \in L^{+}$:

$$
\begin{aligned}
& (\perp+x) \vee(\perp+y)=\perp+(x \vee y) \\
& (\perp+x) \wedge(\perp+y)=\perp+(x \wedge y),
\end{aligned}
$$

where + is defined coordinatewise.

Definition 2 Let $v \in \mathcal{G}(L)$.

(i) $v$ is monotone if for $x, y \in L, x \leq y$ implies $v(x) \leq v(y)$. A bipolar game satisfying monotonicity and such that $v(\top)=1, v(\perp)=-1$ is called a bipolar capacity.

(ii) $v$ is convex if for all $x, y \in L$

$$
v(x \vee y)+v(x \wedge y) \geq v(x)+v(y) .
$$

(iii) $v$ is additive if for any $x$ in $L$

$$
v(x)=v\left(x^{+}\right)+v\left(x^{-}\right)=v^{+}\left(x^{+}\right)+v^{-}\left(\left(x^{-}\right)^{\prime}\right)+v(\perp)
$$

and $v^{+}, v^{-}$are additive multichoice games. Equivalently:

$$
v(x)=\sum_{i \in N} v^{+}\left(\widetilde{x_{i}^{+}}\right)+\sum_{i \in N} v^{-}\left(\widetilde{\left(x_{i}^{-}\right)^{\prime}}\right)+v(\perp), \quad \forall x \in L .
$$

We denote by $\mathcal{A}(L)$ the set of additive bipolar games on $L$.

\section{The core and the Weber set of multichoice games}

We recall in this section main results obtained by the authors for multichoice games [11]. In all this section, $L:=L_{1} \times \cdots L_{n}$, with $L_{i}:=\left\{0,1, \ldots, l_{i}\right\}$ being linear lattices, and $\mathcal{A}(L)$ is the set of additive multichoice games on $L$.

Definition 3 The precore of a multichoice game $v$ on $L$ is defined by

$$
\mathcal{P C}(v):=\{\phi \in \mathcal{A}(L) \mid \phi(x) \geq v(x), \forall x \in L, \text { and } \phi(\top)=v(\top)\} .
$$


The precore, although convex, is unbounded. Indeed, considering a 2-choice game with two players, hence $L:=\{0,1,2\}^{2}$, the conditions on $\phi$ to be element of the precore write:

$$
\begin{aligned}
\phi(2,0)+\phi(0,2) & =v(2,2) \\
\phi(1,0) & \geq v(1,0) \\
\phi(0,1) & \geq v(0,1) \\
\phi(1,0)+\phi(0,1) & \geq v(1,1) .
\end{aligned}
$$

Remark that $\phi(1,0)$ and $\phi(0,1)$ may be taken arbitrarily large. We denote by $\mathcal{P C}^{F}(v):=$ $\operatorname{co}(\operatorname{Ext}(\mathcal{P C}(v)))$ the polytope of $\mathcal{P C}(v)$, where $\operatorname{Ext}(\cdot)$ is the set of extreme points (vertices) of some convex set, and $\mathrm{co}(\cdot)$ is the convex hull of some set.

Definition 4 The core of a multichoice game $v$ on $N$ is defined as:

$$
\begin{aligned}
\mathcal{C}(v):=\{\phi \in \mathcal{A}(L) \mid \phi(x) \geq v(x), \forall x \in L, \\
\left.\quad \text { and } \phi\left(k \wedge l_{1}, \ldots, k \wedge l_{n}\right)=v\left(k \wedge l_{1}, \ldots, k \wedge l_{n}\right), k=1, \ldots, \max _{j} l_{j}\right\} .
\end{aligned}
$$

Remark that the core is a convex bounded set.

We introduce marginal worth vectors $\psi^{C}$ as the vectors of increments along maximal chains $C$ in the lattice $L$. A maximal chain is a sequence $x^{0}:=\perp<x^{1}<\cdots<$ $x^{\sum_{j=1}^{n} l_{j}}:=\top$ of elements of $L$, starting from $\perp$ and reaching $\top$, such that for any $i$, there is no element between $x^{i}$ and $x^{i+1}$. Coordinates of $\psi^{C}$ are denoted by $\psi_{k_{j}}^{C}$, for any player $j \in N$ and any level $k>0$ in $L_{j}$. To any marginal vector is associated an additive game $\phi^{C}$ defined by

$$
\phi_{k_{j}}^{C}:=\sum_{p=1}^{k} \psi_{p_{j}}^{C} .
$$

The important fact is that $\phi=v$ along the chain $C$. The set of all such additive games is called $\mathcal{P} \mathcal{M}(v)$, and the pre-Weber set $\mathcal{P} \mathcal{W}(v)$ is defined as the convex hull of all additive games in $\mathcal{P} \mathcal{M}(v)$. Considering only restricted maximal chains in $L$, i.e., those passing through all $\left(k \wedge l_{1}, \ldots, k \wedge l_{n}\right), k=1, \ldots, \max _{j} l_{j}$, we define $\mathcal{M}(v)$, the set of all additive games $\phi^{C}$ corresponding to marginal worth vectors associated to all restricted maximal chains. Then the Weber set $\mathcal{W}$ is defined as the convex hull of all additive games in $\mathcal{M}(v)$.

The following has been shown.

Proposition 1 Let $v$ be a multichoice games on L. The following holds.

(i) $\mathcal{P C}^{F}(v) \subseteq \mathcal{P W}(v)$

(ii) $\mathcal{C}(v) \subseteq \mathcal{W}(v)$

(iii) If $v$ is convex, then $\mathcal{C}(v)=\mathcal{W}(v)$

(iv) If $v$ is convex, then $\mathcal{P C}^{F}(v)=\mathcal{P W}(v)$. 


\section{The core and the Weber set of bipolar games}

\subsection{Core and precore}

From now on, $L$ is again a product of bipolar lattices. We propose the following definitions.

Definition 5 Let $v$ be a bipolar game. The precore of $v$ is given by:

$$
\begin{aligned}
\mathcal{P C}(v):=\{\phi \in \mathcal{A}(L) \mid \phi(\top) & =v(\top), \phi(\perp)=v(\perp), \\
\phi(x) & \left.=\sum_{i: x_{i}>0} \phi^{+}\left(\widetilde{x_{i}}\right)+\sum_{i: x_{i} \leq 0} \phi^{-}\left(\widetilde{x_{i}^{\prime}}\right)+\phi(\perp) \geq v(x), \quad x \in L\right\}
\end{aligned}
$$

As for multichoice games, it is not difficult to see that the precore is unbounded (see Sec. 3 ), so that we need to add other normalization conditions to keep it bounded.

Definition 6 Let $s:=\max \left(s_{1}, \ldots, s_{n}\right)$ and $t:=\max \left(t_{1}, \ldots, t_{n}\right)$. The core of a bipolar game $v$ is :

$$
\begin{aligned}
\mathcal{C}(v):=\{\phi \in \mathcal{P C}(v) & \mid \phi^{+}\left(t_{1} \wedge k, \ldots, t_{n} \wedge k\right)=v^{+}\left(t_{1} \wedge k, \ldots, t_{n} \wedge k\right), 1 \leq k \leq t \\
& \text { and } \left.\phi^{-}\left(s_{1} \wedge k, \ldots, s_{n} \wedge k\right)=v^{-}\left(s_{1} \wedge k, \ldots, s_{n} \wedge k\right), 1 \leq k \leq s\right\} .
\end{aligned}
$$

Hence, the core of a bipolar game is always a subset of the precore.

Note that the second set of equalities can be rewritten as:

$\phi(((-\perp) \wedge k)+\perp)=\phi\left(\left(s_{1} \wedge k\right)-s_{1}, \ldots,\left(s_{n} \wedge k\right)-s_{n}\right)=v\left(\left(s_{1} \wedge k\right)-s_{1}, \ldots,\left(s_{n} \wedge k\right)-s_{n}\right), 1 \leq k \leq s$, taking into account the fact that $v(\perp)=\phi(\top)$.

Proposition 2 For any bipolar game $v, \mathcal{C}(v)$ and $\mathcal{P C}(v)$ are convex sets, that is, $\forall \phi, \phi^{\prime} \in$ $\mathcal{P C}(v), \forall \lambda \in[0,1], \lambda \phi+(1-\lambda) \phi^{\prime} \in \mathcal{P C}(v)$, and similarly for $\mathcal{C}(v)$.

Proof: $\quad$ Let $\phi_{1}, \phi_{2} \in \mathcal{P C}(v), \lambda \in[0,1]$ and $\phi=\lambda \phi_{1}+(1-\lambda) \phi_{2}$. Then

$$
\begin{aligned}
\phi(x) & =\lambda \phi_{1}(x)+(1-\lambda) \phi_{2}(x) \geq \lambda v(x)+(1-\lambda) v(x)=v(x) \\
\phi(\top) & =\lambda \phi_{1}(\top)+(1-\lambda) \phi_{2}(\top)=\lambda v(\top)+(1-\lambda) v(\top)=v(\top) \\
\phi(\perp) & =\lambda \phi_{1}(\perp)+(1-\lambda) \phi_{2}(\perp)=\lambda v(\perp)+(1-\lambda) v(\perp)=v(\perp)
\end{aligned}
$$

and $\phi(0)=0$. Since $\phi_{1}, \phi_{2} \in \mathcal{A}(L)$, we have $\phi \in \mathcal{A}(L)$. Thus $\phi \in \mathcal{P C}(v)$.

Now, let $\phi_{1}, \phi_{2} \in \mathcal{C}(v)$. For all $0 \leq k \leq s, \lambda \in[0,1]$,

$$
\begin{aligned}
\phi & \left(\left(s_{1} \wedge k\right)-s_{1}, \ldots,\left(s_{n} \wedge k\right)-s_{n}\right) \\
& =\lambda \phi_{1}\left(\left(s_{1} \wedge k\right)-s_{1}, \ldots,\left(s_{n} \wedge k\right)-s_{n}\right)+(1-\lambda) \phi_{2}\left(\left(s_{1} \wedge k\right)-s_{1}, \ldots,\left(s_{n} \wedge k\right)-s_{n}\right) \\
& =\lambda v\left(s_{1} \wedge k-s_{1}, \ldots, s_{n} \wedge k-s_{n}\right)+(1-\lambda) v\left(s_{1} \wedge k-s_{1}, \ldots, s_{n} \wedge k-s_{n}\right) \\
& =v\left(s_{1} \wedge k-s_{1}, \ldots, s_{n} \wedge k-s_{n}\right), k=1, \ldots, s .
\end{aligned}
$$

Similarly, $\phi\left(t_{1} \wedge k, \ldots, t_{n} \wedge k\right)=v\left(t_{1} \wedge k, \ldots, t_{n} \wedge k\right), k=1, \ldots, t$. Since $\phi_{1}, \phi_{2} \in \mathcal{C}(v) \subseteq$ $\mathcal{P C}(v)$, we deduce $\phi \in \mathcal{P C}(v)$. Thus $\phi \in \mathcal{C}(v)$. 
Proposition 3 Let $v$ be a bipolar game. Then $\phi=\left(\phi^{+}, \phi^{-}\right) \in \mathcal{P C}(v)$ implies $\phi^{+} \in$ $\mathcal{P C}\left(v^{+}\right)$and $\phi^{-} \in \mathcal{P C}\left(v^{-}\right)$, and similarly for $\mathcal{C}(v)$. Hence, $\mathcal{P C}(v) \neq \emptyset \Rightarrow \mathcal{P C}\left(v^{+}\right), \mathcal{P C}\left(v^{-}\right) \neq$ $\emptyset$, and similarly for the core.

Be careful that in the above, $\mathcal{P C}\left(v^{+}\right), \mathcal{P C}\left(v^{-}\right)$are precores of multichoice games (see Def. $3)$.

Proof: Let $\phi=\left(\phi^{+}, \phi^{-}\right) \in \mathcal{P C}(v)$. This implies that $\phi^{+}, \phi^{-}$are additive, and applying (2):

$$
\begin{aligned}
\phi(\top) & =\phi^{+}(\top)=v(\top)=v^{+}(\top) \\
\phi(\perp) & =-\phi^{-}(-\perp)=v(\perp)=-v^{-}(-\perp) \\
\phi(x) & =\phi^{+}\left(x^{+}\right)+\phi^{-}\left(\left(x^{-}\right)^{\prime}\right)+\phi(\perp) \geq v(x), \quad \forall x \in L .
\end{aligned}
$$

Restricting (10) to $L^{+}$and $L^{-}$respectively, we get:

$$
\begin{aligned}
& \phi(x)=\phi^{+}(x) \geq v(x)=v^{+}(x), \quad \forall x \in L^{+} \\
& \phi(x)=\phi^{-}\left(x^{\prime}\right)+\phi(\perp) \geq v(x)=v^{-}\left(x^{\prime}\right)+v(\perp), \quad \forall x \in L^{-} .
\end{aligned}
$$

Let us show that $\phi^{+} \in \mathcal{P C}\left(v^{+}\right)$. We know already that $\phi^{+}$is an additive multichoice game. By (8) and (11), we deduce $\phi^{+}(\top)=v^{+}(\top)$ and $\phi^{+}(x) \geq v^{+}(x)$ for all $x \in L^{+}$. Hence $\phi^{+} \in \mathcal{P C}\left(v^{+}\right)$. Similarly, $\phi^{-}$is an additive multichoice game, and by (9) and (12), we deduce $\phi^{-}(-\perp)=v^{-}(-\perp)$ and $\phi^{-}\left(x^{\prime}\right) \geq v^{-}\left(x^{\prime}\right)$ for all $x \leq \mathbb{O}$. Hence $\phi^{-} \in \mathcal{P C}\left(v^{-}\right)$.

It remains to show the case of the core. Let $\phi=\left(\phi^{+}, \phi^{-}\right) \in \mathcal{C}(v)$. Then

$$
\begin{aligned}
\phi\left(t_{1} \wedge k, \ldots, t_{n} \wedge k\right) & =v\left(t_{1} \wedge k, \ldots, t_{n} \wedge k\right), \quad 1 \leq k \leq t \\
\phi\left(\left(s_{1} \wedge k\right)-s_{1}, \ldots,\left(s_{n} \wedge k\right)-s_{n}\right) & =v\left(\left(s_{1} \wedge k\right)-s_{1}, \ldots,\left(s_{n} \wedge k\right)-s_{n}\right), \quad 0 \leq k \leq s
\end{aligned}
$$

Then clearly $\phi^{+}\left(t_{1} \wedge k, \ldots, t_{n} \wedge k\right)=v^{+}\left(t_{1} \wedge k, \ldots, t_{n} \wedge k\right), 1 \leq k \leq t$, which proves $\phi^{+} \in \mathcal{C}\left(v^{+}\right)$, and $\phi^{-}\left(s_{1} \wedge k, \ldots, s_{n} \wedge k\right)=\phi\left(\left(s_{1} \wedge k\right)-s_{1}, \ldots,\left(s_{n} \wedge k\right)-s_{n}\right)-\phi(\perp)=$ $v\left(\left(s_{1} \wedge k\right)-s_{1}, \ldots,\left(s_{n} \wedge k\right)-s_{n}\right)-v(\perp)=v^{-}\left(s_{1} \wedge k, \ldots, s_{n} \wedge k\right), 0 \leq k \leq s$, which proves $\phi^{-} \in \mathcal{C}\left(v^{-}\right)$.

The converse is not true since choosing two multichoice games $v^{+}, v^{-}$does not determine uniquely a bipolar game $v$, since $v(x)$ is not determined for all $x$ having both positive and negative coordinates, and so can be taken arbitrarily large. The converse becomes true if $v$ is convex, as shown in Sec. 4.3.

\subsection{Core and balancedness}

Using concepts from linear programming, we give sufficient and necessary conditions of nonemptiness of the precore and core of a bipolar game.

Definition 7 (i) A collection $\mathcal{B}$ of elements of $L \backslash\{\mathbb{O}\}$ is prebalanced if it exist positive coefficients $\mu(x), x \in \mathcal{B}$, such that $\sum_{x: x_{i}=t_{i}} \mu(x)=1, \forall i \in N, \sum_{x: x_{i}=-s_{i}} \mu(x)=1$, $\forall i \in N$, and $\sum_{x: x_{i}=k} \mu(x)=0, k=-s_{i}+1, \ldots,-1,1, \ldots, t_{i}-1, i \in N$. 
(ii) A bipolar game $v$ is prebalanced if for every prebalanced collection $\mathcal{B}$ of elements of $L \backslash\{\mathbb{O}\}$ with coefficients $\mu(x), x \in \mathcal{B}$, it holds

$$
\sum_{x \in \mathcal{B}} \mu(x) v(x) \leq v(\top)+v(\perp)
$$

Proposition 4 A bipolar game has a nonempty precore if and if only it is prebalanced.

Proof: Consider the following linear programming problem with variables $\phi^{+}\left(\widetilde{k_{i}}\right), k=$ $1, \ldots, t_{i}, \phi^{-}\left(\widetilde{k}_{i}\right), k=1, \ldots, s_{i}, i \in N$ :

$$
\begin{aligned}
\operatorname{minimize} & z=\sum_{i \in N} \phi^{+}\left(\widetilde{t_{i}}\right)-\sum_{i \in N} \phi^{-}\left(\widetilde{s_{i}}\right) \\
\text { under } & \sum_{x_{i}>0} \phi^{+}\left(\widetilde{x_{i}}\right)+\sum_{x_{i} \leq 0} \phi^{-}\left(\widetilde{x_{i}^{\prime}}\right)-\sum_{i \in N} \phi^{-}\left(\widetilde{s_{i}}\right) \geq v(x), \forall x \in L .
\end{aligned}
$$

Remarking that $z=\phi(\top)+\phi(\mathbb{O})+\phi(\perp)$, and that the constraints write $\phi(x) \geq v(x)$, the precore is nonempty if and only if the optimal solution is such that:

$$
z^{*} \leq v(\top)+v(\perp) .
$$

Now, the dual problem is

$$
\begin{aligned}
& \operatorname{maximize} w=\sum_{x \in L} \mu(x) v(x) \\
& \text { under } \mu(x) \geq 0, \forall x \in L, \\
& \quad \sum_{x_{-i} \in L_{-i}} \mu\left(t_{i}, x_{-i}\right)=1, \forall i \in N \\
& \quad \sum_{x \in L: x_{i}<0} \mu(x)=1, \forall i \in N \\
& \quad \text { and } \sum_{x: x_{i}=k} \mu(x)=0, k=-s_{i}+1, \ldots,-1,1, \ldots, t_{i}-1, \forall i \in N .
\end{aligned}
$$

By the duality theorem, the dual problem has a solution iff the primal problem has a solution, and in this case $w^{*}=z^{*}$. Moreover, the two last sets of constraints imply

$$
\sum_{x: x_{i}=-s_{i}} \mu(x)=1, \forall i \in N
$$

hence the desired result.

We define balancedness of bipolar game as follows.

Definition 8 (i) A collection $\mathcal{B}$ of elements of $L \backslash\{\mathbb{O}\}$ is balanced if it exist positive coefficients $\mu(x), x \in \mathcal{B}$, such that $\sum_{x: x_{i}=t_{i}} \mu(x)=t-t_{i}+1, \sum_{x: x_{i}=-s_{i}} \mu(x)=1$, $\sum_{x: x_{i}=k_{i}} \mu(x)=1$, for all $i \in N, k=-s_{i}+1, \ldots,-1,1, \ldots, t_{i}-1$. 
(ii) A bipolar game $\nu$ is balanced if for every balanced collection $\mathcal{B}$ of elements of $L \backslash\{\mathbb{O}\}$ with coefficients $\mu(x), x \in \mathcal{B}$, it holds

$$
\sum_{x \in \mathcal{B}} \mu(x) v(x) \leq \sum_{k=1}^{t} v\left(k \wedge t_{1}, \ldots, k \wedge t_{n}\right)+\sum_{k=0}^{s-1} v\left(\left(k \wedge s_{1}\right)-s_{1}, \ldots,\left(k \wedge s_{n}\right)-s_{n}\right) .
$$

Proposition 5 A bipolar game has a nonempty core if and if only it is balanced.

Proof: Consider the following linear programming problem with variables $\phi^{+}\left(\tilde{k}_{i}\right), 1 \leq$ $k \leq t_{i}$, and $\phi^{-}\left(\tilde{k}_{i}\right), 1 \leq k \leq s_{i}, i \in N$ :

$$
\begin{gathered}
\text { minimize } z=\sum_{k=1}^{t} \sum_{i \in N} \phi^{+}\left(\widetilde{k \wedge t_{i}}\right)+\sum_{k=0}^{s-1} \sum_{i \in N} \phi^{-}\left(\widetilde{k \wedge s_{i}}\right)-s \sum_{i \in N} \phi^{-}\left(\widetilde{s_{i}}\right) \\
\text { under } \sum_{x_{i}>0} \phi^{+}\left(\widetilde{x_{i}}\right)+\sum_{x_{i} \leq 0} \phi^{-}\left(\widetilde{x_{i}^{\prime}}\right)-\sum_{i \in N} \phi^{-}\left(\widetilde{s_{i}}\right) \geq v(x), \forall x \in L .
\end{gathered}
$$

Rewriting $z$ in terms of $\phi$ leads to

$$
z=\sum_{k=1}^{t} \phi\left(k \wedge t_{1}, \ldots, k \wedge t_{n}\right)+\sum_{k=0}^{s-1} \phi\left(\left(k \wedge s_{1}\right)-s_{1}, \ldots,\left(k \wedge s_{n}\right)-s_{n}\right) .
$$

Remarking in addition that the constraints write $\phi(x) \geq v(x)$ for all $x \in L$, the core is nonempty if and only if the optimal solution is such that

$$
z^{*} \leq \sum_{k=1}^{t} v\left(k \wedge t_{1}, \ldots, k \wedge t_{n}\right)+\sum_{k=0}^{s-1} v\left(\left(k \wedge s_{1}\right)-s_{1}, \ldots,\left(k \wedge s_{n}\right)-s_{n}\right) .
$$

Now, observe that $z$ can be rewritten as

$$
z=\sum_{i \in N} \phi^{+}\left(\widetilde{t_{i}}\right)\left(t-t_{i}+1\right)+\sum_{i \in N} \sum_{1 \leq k<t_{i}} \phi^{+}\left(\widetilde{k_{i}}\right)-\sum_{i \in N} s_{i} \phi^{-}\left(\widetilde{s}_{i}\right)+\sum_{i \in N} \sum_{1 \leq k<s_{i}} \phi^{-}\left(\widetilde{k}_{i}\right)
$$

Hence, the dual problem writes:

$$
\begin{aligned}
& \operatorname{maximize} w=\sum_{x \in L} \mu(x) v(x) \\
& \text { under } \mu(x) \geq 0, \forall x \in L, \\
& \quad \sum_{x_{-i} \in L_{-i}} \mu\left(t_{i}, x_{-i}\right)=t-t_{i}+1 \forall i \in N \\
& \quad \sum_{x \in L: x_{i}<0} \mu(x)=s_{i}, \forall i \in N \\
& \sum_{x \in L: x_{i}=k} \mu(x)=1, k=-s_{i}+1, \ldots,-1,1, \ldots, t_{i}-1, i \in N .
\end{aligned}
$$

By the duality theorem, the dual problem has a solution iff the primal problem has a solution, and in this case $w^{*}=z^{*}$. Moreover, the two last sets of constraints imply

$$
\sum_{x \in L: x_{i}=-s_{i}} \mu(x)=1, \quad \forall i \in N,
$$

hence the desired result. 


\subsection{Core and convexity}

Proposition 6 If $v$ is a convex bipolar game, then

(i) $v^{-}, v^{+}$are convex games

(ii) $\mathcal{P C}\left(v^{+}\right), \mathcal{P C}\left(v^{-}\right), \mathcal{C}\left(v^{+}\right), \mathcal{C}\left(v^{-}\right) \neq \emptyset$

(iii) $\mathcal{P C}(v), \mathcal{C}(v) \neq \emptyset$. Moreover, $\mathcal{P C}(v)=\left\{\phi=\left(\phi^{+}, \phi^{-}\right) \in \mathcal{A}(L) \mid \phi^{+} \in \mathcal{P C}\left(v^{+}\right), \quad \phi^{-} \in\right.$ $\left.\mathcal{P C}\left(v^{-}\right)\right\}$, and similarly for $\mathcal{C}(v)$.

\section{Proof:}

(i) First we prove $v^{+}$is convex : If $v$ is convex, $v(x)+v(y) \leq v(x \vee y)+v(x \wedge y)$. If $x, y \geq \mathbb{O}$, then $x \wedge y, x \vee y \geq \mathbb{O}$. Hence $v^{+}(x)+v^{+}(y) \leq v^{+}(x \vee y)+v^{+}(x \wedge y)$, i.e. $v^{+}$is convex.

Now we prove that $v^{-}$is convex. For $x, y \leq \mathbb{O}$ :

$$
\begin{aligned}
v^{-}\left(x^{\prime}\right)+v^{-}\left(y^{\prime}\right) & =v(x)-v(\perp)+v(y)-v(\perp) \\
& \leq v(x \vee y)+v(x \wedge y)-2 v(\perp) \\
& =v\left(\left(x^{\prime}+\perp\right) \vee\left(y^{\prime}+\perp\right)\right)+v\left(\left(x^{\prime}+\perp\right) \wedge\left(y^{\prime}+\perp\right)\right)-2 v(\perp) \\
& =v\left(\left(x^{\prime} \vee y^{\prime}\right)+\perp\right)+v\left(\left(x^{\prime} \wedge y^{\prime}\right)+\perp\right)-2 v(\perp) \text { by }(4) \text { and }(5) \\
& =v^{-}\left[\left(\left(x^{\prime} \vee y^{\prime}\right)+\perp\right)^{\prime}\right]+v^{-}\left[\left(\left(x^{\prime} \wedge y^{\prime}\right)+\perp\right)^{\prime}\right] \\
& =v^{-}\left(x^{\prime} \vee y^{\prime}\right)+v^{-}\left(x^{\prime} \wedge y^{\prime}\right) .
\end{aligned}
$$

Therefore $v^{-}$is convex.

(ii) By (i), we know that $v^{-}, v^{+}$are two multichoice convex games, then by Prop. 1 , $\mathcal{P C}\left(v^{+}\right), \mathcal{P C}\left(v^{-}\right), \mathcal{C}\left(v^{+}\right), \mathcal{C}\left(v^{-}\right) \neq \emptyset$.

(iii) First we prove that $\mathcal{P C}(v)=\left\{\left(\phi^{+}, \phi^{-}\right) \mid \phi^{+} \in \mathcal{P C}\left(v^{+}\right), \phi^{-} \in \mathcal{P C}\left(v^{-}\right)\right\}$. By Prop. 3, it remains to show that any $\left(\phi^{+}, \phi^{-}\right)$is element of $\mathcal{P C}(v)$. Let $\phi^{+} \in \mathcal{P C}\left(v^{+}\right), \phi^{-} \in$ $\mathcal{P C}\left(v^{-}\right)$and put $\phi(x):=\phi^{+}\left(x^{+}\right)+\phi^{-}\left(x^{-\prime}\right)+v(\perp)$. Then $\phi(\mathbb{O})=\phi^{+}(\mathbb{O})+\phi^{-}\left(\mathbb{O}^{\prime}\right)+$ $v(\perp)=\phi^{-}\left(\mathbb{O}^{\prime}\right)+v(\mathbb{O})-v^{-}\left(\mathbb{O}^{\prime}\right)=v(\mathbb{O})=0, \phi(\top)=\phi^{+}(\top)+\phi^{-}\left(\mathbb{O}^{\prime}\right)+v(\perp)=v(\top)+$ $v(\mathbb{O})-v(\perp)+v(\perp)=v(\top), \phi(\perp)=\phi^{+}(\mathbb{O})+\phi^{-}\left(\perp^{\prime}\right)+v(\perp)=\phi^{-}(\mathbb{O})+v(\perp)=v(\perp)$.

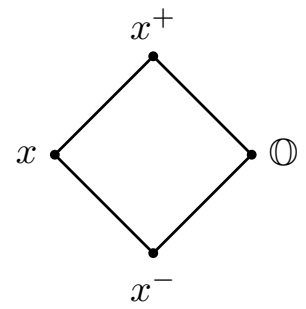

Since $v$ is convex, $v\left(x^{+}\right)+v\left(x^{-}\right) \geq v(x)+v(\mathbb{O})$. Hence,

$$
\begin{aligned}
\phi(x) & =\phi^{+}\left(x^{+}\right)+\phi^{-}\left(x^{-\prime}\right)+v(\perp) \\
& \geq v^{+}\left(x^{+}\right)+v^{-}\left(x^{-\prime}\right)+v(\perp) \\
& =v\left(x^{+}\right)+v\left(x^{-}\right) \\
& \geq v(x)+v(\mathbb{O}) \\
& =v(x)
\end{aligned}
$$


$\Rightarrow \phi \in \mathcal{P C}(v)$.

Now we prove $\mathcal{C}(v) \neq \emptyset$ : If $\phi^{+} \in \mathcal{C}\left(v^{+}\right), \phi^{-} \in \mathcal{C}\left(v^{-}\right)$, then $\phi^{+}, \phi^{-}$satisfies also the following properties : $\phi\left(k \wedge t_{1}, \ldots, k \wedge t_{n}\right)=\phi^{+}\left(k \wedge t_{1}, \ldots, k \wedge t_{n}\right)+\phi^{-}\left(\mathbb{O}^{\prime}\right)+v(\perp)=$ $v^{+}\left(k \wedge t_{1}, \ldots, k \wedge t_{n}\right)=v\left(k \wedge t_{1}, \ldots, k \wedge t_{n}\right), \forall 1 \leq k \leq t$ and $\phi((-\perp) \wedge k+\perp)=$ $\phi^{+}(\mathbb{O})+\phi^{-}((-\perp) \wedge k)+v(\perp)=v^{-}((-\perp) \wedge k)+v((-\perp) \wedge k+\perp)-v^{-}((-\perp) \wedge k)=$ $v((-\perp) \wedge k+\perp), \forall 0 \leq k \leq s$. Hence $\phi \in \mathcal{C}(v)$.

\subsection{The core and the Weber set}

As for multichoice games (see Sec. 3), we may introduce the marginal worth vector $\psi^{C}$ along a maximal chain $C$ in $L$. Since eventually, only the corresponding additive game $\phi^{C}$ is of interest, we consider only the latter.

Definition 9 Let $C=\left\{\perp=x^{-\sum_{i=1}^{n} s_{i}}, \ldots, x^{-1}, x^{0}=0, x^{+1}, \ldots, \top=x^{+\sum_{i=1}^{n} t_{i}}\right\}$ a maximal chain of $L$ from $\perp$ to $\top$ passing through $\mathbb{O}$. To $C$ we make correspond the additive bipolar game $\phi$ unambigously defined by $\phi\left(x^{i}\right)=v\left(x^{i}\right), i=-\sum_{i=1}^{n} s_{i}, \ldots,-1,0,1, \ldots, \sum_{i=1}^{n} t_{i}$. We denote by $\mathcal{P} \mathcal{M}(v)$ the set of such additive bipolar games.

We set $s:=\max \left(s_{1}, \ldots, s_{n}\right)$ and $t:=\max \left(t_{1}, \ldots, t_{n}\right)$ as before.

Definition $10 A$ restricted maximal chain $C_{r}$ is a maximal chain passing through all points $\left(\left(s_{1} \wedge k\right)-s_{1}, \ldots,\left(s_{n} \wedge k\right)-s_{n}\right), 0 \leq k \leq s$, and $\left(t_{1} \wedge k, \ldots, t_{n} \wedge k\right), 1 \leq k \leq t$. We denote by $\mathcal{M}(v)$ the set of additive bipolar games generated by all restricted maximal chains.

Proposition 7 If $v$ is convex, then

(i) $\mathcal{P} \mathcal{M}(v) \subseteq \mathcal{P C}(v)$

(ii) $\mathcal{M}(v) \subseteq \mathcal{C}(v)$

Proof: Any maximal chain $C$ passing through $\mathbb{O}$ can be decomposed into two maximal chains $C^{+}, C^{-}$in $L^{+},-L^{-}$respectively. Since $v^{+}, v^{-}$are convex by Prop. $6, C^{+}, C^{-}$ generate two additive multichoice games $\phi^{+}, \phi^{-}$coinciding with $v^{+}, v^{-}$, which belong to the precores of $v^{+}, v^{-}$respectively. Now, by Prop. 3, we know that $\left(\phi^{+}, \phi^{-}\right) \in \mathcal{P C}(v)$.

If $C$ is a restricted chain, then $C^{+}, C^{-}$in $L^{+},-L^{-}$are restricted chains too, and by the same reasoning they generate an element of the core of $v$.

We define the pre-Weber and Weber sets as follows.

Definition 11 The pre-Weber set of a bipolar game $v$, denoted by $\mathcal{P W}(v)$, is the convex hull of $\mathcal{P} \mathcal{M}(v)$, denoted by $\mathcal{P} \mathcal{W}(v)$. The Weber set of $v$, denoted by $\mathcal{W}(v)$, is the convex hull of $\mathcal{M}(v)$.

From Prop. 7 and the convexity of core and precore (Prop. 2), we deduce the following. 
Corollary 1 If $v$ is a convex bipolar game, then $\mathcal{P W}(v) \subseteq \mathcal{P C}(v)$ and $\mathcal{W}(v) \subseteq \mathcal{C}(v)$.

Theorem 1 Let $v$ be a convex bipolar game. Then

(i) $\mathcal{P} \mathcal{M}(v)$ is the set of vertices of $\mathcal{P C}(v)$.

(ii) $\mathcal{M}(v)$ is the set of vertices of $\mathcal{C}(v)$.

Proof: Let $\phi \in \mathcal{C}(v)$, then $\phi^{+} \in \mathcal{C}\left(v^{+}\right), \phi^{-} \in \mathcal{C}\left(v^{-}\right)$. Games $\phi^{+}$and $\phi^{-}$are vertices of the precore of $v^{+}$and $v^{-}$if and only if $\phi$ is a vertice of the core of $v$. We know by Prop. 1 that the set $\mathcal{P M}$ of a convex multichoice game is the set of vertices of its precore. Thus, $\mathcal{P M}(v)$ is the set of vertices of $\mathcal{P C}(v)$. The proof is similar for the core.

The following result concerns the core and the bounded part of the precore, and extends Th. 1 (i) (ii).

Theorem 2 Let $v$ be a bipolar game. Then

(i) $\mathcal{P C}^{F}(v) \subseteq \mathcal{P} \mathcal{W}(v)$

(ii) $\mathcal{C}(v) \subseteq \mathcal{W}(v)$

Proof: By Prop. 1 (i) and (ii), we have $\mathcal{P C}^{F}\left(v^{+}\right) \subseteq \mathcal{P} \mathcal{W}\left(v^{+}\right), \mathcal{P} \mathcal{C}^{F}\left(v^{-}\right) \subseteq \mathcal{P} \mathcal{W}\left(v^{-}\right)$and $\mathcal{C}\left(v^{+}\right) \subseteq \mathcal{W}\left(v^{+}\right), \mathcal{C}\left(v^{-}\right) \subseteq \mathcal{W}\left(v^{-}\right)$

(i) Let us prove $\mathcal{P C}^{F}(v) \subseteq \mathcal{P W}(v)$. Let $\phi \in \mathcal{P C}^{F}(v)$, then $\phi^{+} \in \mathcal{P C}^{F}\left(v^{+}\right)$and $\phi^{-} \in \mathcal{P C}^{F}\left(v^{-}\right)$. Hence $\phi^{+} \in \mathcal{P} \mathcal{W}\left(v^{+}\right)$and $\phi^{-} \in \mathcal{P} \mathcal{W}\left(v^{-}\right)$. Thus $\phi \in \mathcal{P} \mathcal{W}(v)$.

(ii) Works similarly for $\mathcal{C}(v) \subseteq \mathcal{W}(v)$.

We immediately deduce the following.

Corollary 2 Let $v$ be a convex bipolar game, then

(i) if $\exists i \in N$ s.t. $s_{i}>1$ or $t_{i}>1$, then $\mathcal{P C}$ is unbounded, and $\mathcal{P W}(v)=\mathcal{P C}^{F}(v)$;

(ii) $\mathcal{W}(v)=\mathcal{C}(v)$.

\subsection{A remark on additivity}

For classical games, additivity appears as a special case of convexity. Namely, the additivity condition writes $v(A \cup B)=v(A)+v(B)$ for any $A, b$ such that $A \cap B=\emptyset$, while convexity is $v(A \cup B) \geq v(A)+v(B)-v(A \cap B)$. Our definition of additivity in Section 2 does not follow this line, but corresponds to the condition $v(A)=\sum_{i \in A} v(\{i\})$, equivalent to the above condition of additivity in the classical case. We show that for the bipolar case, they are no more equivalent, and point out the reason. 
Let us consider $x, y \in L$ such that $x \wedge y=\perp$. This amounts to $x^{+} \wedge y^{+}=\mathbb{O}$, and $x^{-} \wedge y^{-}=\perp$, and consequently $\left(x^{-} \wedge y^{-}\right)^{\prime}=\left(x^{-}\right)^{\prime} \wedge\left(y^{-}\right)^{\prime}=\mathbb{O}$. Let us assume that $v$ is a bipolar additive game as defined in Sec. 2, and verify if $v(x \vee y)=v(x)+v(y)$.

$$
\begin{aligned}
v(x \vee y) & =v^{+}\left((x \vee y)^{+}\right)+v^{-}\left(\left((x \vee y)^{-}\right)^{\prime}\right)+v(\perp) \\
& =v^{+}\left(x^{+} \vee y^{+}\right)+v^{-}\left(\left(x^{-}\right)^{\prime} \vee\left(y^{-}\right)^{\prime}\right)+v(\perp) \\
& =v^{+}\left(x^{+}\right)+v^{+}\left(y^{+}\right)+v^{-}\left(\left(x^{-}\right)^{\prime}\right)+v^{-}\left(\left(y^{-}\right)^{\prime}\right)+v(\perp) \\
& =v(x)+v(y)-v(\perp) .
\end{aligned}
$$

Clearly, the two definitions are not equivalent. The reason is the normalization condition $v(\mathbb{O})=0=v^{+}(\mathbb{O})+v^{-}\left(\mathbb{O}^{\prime}\right)+v(\perp)=v^{+}(\mathbb{O})+v^{-}(-\perp)+v(\perp)$, which makes the term $v(\perp)$ necessary.

\section{Application to bicooperative games and bicapaci- ties}

We shortly describe the case of bicooperative games and bicapacities, and apply the above general results to this case.

As explained in the introduction, we use here the notation $\mathcal{Q}(N)$ for the set of bicoalitions. We define the order relation $\sqsubseteq$ on $\mathcal{Q}(N)$ by

$$
(S, T) \sqsubseteq\left(S^{\prime}, T^{\prime}\right) \Leftrightarrow S \subseteq S^{\prime}, T \supseteq T^{\prime}
$$

Doing so, $(\mathcal{Q}(N), \sqsubseteq)$ is a lattice with supremum and infimum defined by:

$$
\begin{aligned}
& (S, T) \sqcup\left(S^{\prime}, T^{\prime}\right):=\left(S \cup S^{\prime}, T \cap T^{\prime}\right) \\
& (S, T) \sqcap\left(S^{\prime}, T^{\prime}\right):=\left(S \cap S^{\prime}, T \cup T^{\prime}\right) .
\end{aligned}
$$

(see [8]). $(\mathcal{Q}(N), \sqsubseteq)$ is also a product of bipolar linear lattices $\{-1,0,1\}$, as already mentionned in Sec. 2. A bicooperative game [1] is a function $v: \mathcal{Q}(N) \rightarrow \mathbb{R}$ such that $v(\emptyset, \emptyset)=0$. A bicapacity [6] is a monotonic bicooperative game, i.e., $v(S, T) \leq v\left(S^{\prime}, T^{\prime}\right)$ whenever $(S, T) \sqsubseteq\left(S^{\prime}, T^{\prime}\right)$, and such that $v(N, \emptyset)=1$ and $v(\emptyset, N)=-1$.

Using these specific notations, let us define convex and additive bicooperative games, as well as subsequent notions.

- Eq. (1) rewrites as follows: for any $(\emptyset, T) \in \mathcal{Q}(N),(\emptyset, T)^{\prime}=(N \backslash T, \emptyset)$. Hence, $v^{-}(T, \emptyset)=v(\emptyset, N \backslash T)-v(\emptyset, N)$, and $v^{-}(N, \emptyset)=-v(\emptyset, N)$. We could consider for simplicity that $v^{+}, v^{-}$are classical games on $N$, dropping the $\emptyset$ argument. If $v^{-}$is additive, then $v^{-}(N \backslash T)=-v(\emptyset, N)-v^{-}(T)$.

- The above remarks permit to write: $v$ is additive if $v(S, T)=v^{+}(S)-v^{-}(T)$, where $v^{+}, v^{-}$are two additive classical games, defined by $v^{+}(S)=v(S, \emptyset)$, and $v^{-}(T)=-v(\emptyset, T)$. Let us denote by $\mathcal{A}(\mathcal{Q}(N))$ the set of additive bicooperative games.

- $v$ is convex if $v\left(S \cup S^{\prime}, T \cap T^{\prime}\right)+v\left(S \cap S^{\prime}, T \cup T^{\prime}\right) \geq v(S, T)+v\left(S^{\prime}, T^{\prime}\right)$. 
- The core of $v$ is defined by

$$
\begin{aligned}
\mathcal{C}(v):=\{\phi \in \mathcal{A}(\mathcal{Q}(N)) \mid \phi(S, T) \geq v(S, T), \forall(S, T) \in \mathcal{Q}(N), \\
\left.\phi^{+}(N)=v(N, \emptyset), \phi^{-}(N)=-v(\emptyset, N)\right\} .
\end{aligned}
$$

- A collection $\mathcal{B}$ of elements of $\mathcal{Q}(N) \backslash(\emptyset, \emptyset)$ is balanced if it exist positive coefficients $\mu(S, T),(S, T) \in \mathcal{B}$, such that $\sum_{S \ni i} \mu(S, T)=1$ for all $i \in N$, and $\sum_{T \ni i} \mu(S, T)=1$ for all $i \in N$.

- A bicooperative game $v$ is balanced if for every balanced collection $\mathcal{B}$ of elements of $\mathcal{Q}(N) \backslash(\emptyset, \emptyset)$ with coefficients $\mu(S, T),(S, T) \in \mathcal{B}$, it holds

$$
\sum_{(S, T) \in \mathcal{B}} \mu(S, T) v(S, T) \leq v(N, \emptyset)+v(\emptyset, N)
$$

- Let $C$ be a maximal chain in $\mathcal{Q}(N)$, from $(\emptyset, N)$ to $(N, \emptyset)$ and passing through $(\emptyset, \emptyset)$. To $C$ we associate the additive bicooperative game $\phi_{C}$ by:

$$
\begin{array}{ll}
\phi_{C}^{+}(i):=v\left((S, T)^{i+}\right)-v\left(p\left((S, T)^{i+}\right)\right), & i \in N \\
\phi_{C}^{-}(i):=v\left((S, T)^{i-}\right)-v\left(p\left((S, T)^{i-}\right)\right), & i \in N
\end{array}
$$

where $(S, T)_{i+}$ (resp. $\left.(S, T)_{i+}\right)$ is the first element in $C$ such that $S \ni i$ (resp. $T \not \ngtr i)$, and $p(S, T)$ is the element preceding $(S, T)$ in $C$. Then $\phi_{C}=v$ on $C$. The set of all such additive games is denoted by $\mathcal{M}(v)$, and the Weber set $\mathcal{W}(v)$ is the convex hull of $\mathcal{M}(v)$.

The definition of additivity coincides with the one given originally in [7] for bicapacities. Note that the precore and core definitions collapse into a single one, since there is only one level above and below 0 .

The results shown in Section 4 all holds, with the above definitions. We summarize them in the next proposition.

Proposition 8 Let $v$ be a bicooperative game. The following holds.

(i) The core is a convex nonemptyset if and only if $v$ is balanced.

(ii) If $v$ is convex, the core is non empty and contains all additive bicooperative games $\phi$ such that $\phi^{+}, \phi^{-}$are in the cores of $v^{+}, v^{-}$respectively and only these ones.

(iii) $\mathcal{C}(v) \subseteq \mathcal{W}(v)$

(iv) If $v$ is convex, then $\mathcal{C}(v)=\mathcal{W}(v)$.

\section{References}

[1] J.M. Bilbao. Cooperative games on combinatorial structures. Kluwer Academic Publishers, 2000. 
[2] G. Choquet. Theory of capacities. Annales de l'Institut Fourier, 5:131-295, 1953.

[3] B.A. Davey and H.A. Priestley. Introduction to Lattices and Orders. Cambridge University Press, 1990.

[4] D. Felsenthal and M. Machover. Ternary voting games. Int. J. of Game Theory, 26:335-351, 1997.

[5] M. Grabisch. An axiomatization of the Shapley value and interaction index for games on lattices. In SCIS-ISIS 2004, 2nd Int. Conf. on Soft Computing and Intelligent Systems and 5th Int. Symp. on Advanced Intelligent Systems, Yokohama, Japan, September 2004.

[6] M. Grabisch and Ch. Labreuche. Bi-capacities for decision making on bipolar scales. In EUROFUSE Workshop on Informations Systems, pages 185-190, Varenna, Italy, September 2002.

[7] M. Grabisch and Ch. Labreuche. Capacities on lattices and $k$-ary capacities. In $3 d$ Int, Conf. of the European Soc. for Fuzzy Logic and Technology (EUSFLAT 2003), pages 304-307, Zittau, Germany, September 2003.

[8] M. Grabisch and Ch. Labreuche. Bi-capacities. Part I: definition, Möbius transform and interaction. Fuzzy Sets and Systems, 151:211-236, 2005.

[9] M. Grabisch and Ch. Labreuche. Bi-capacities. Part II: the Choquet integral. Fuzzy Sets and Systems, 151:237-259, 2005.

[10] M. Grabisch and F. Lange. Games on lattices, multichoice games and the Shapley value: a new approach. Mathematical Methods of Operations Research, submitted.

[11] M. Grabisch and L. Xie. A new investigation about the core and Weber set of multichoice games. Int. J. of Game Theory, submitted.

[12] C.R Hsiao and T.E.S. Raghavan. Shapley value for multichoice cooperative games, I. Games and Economic Behavior, 5:240-256, 1993.

[13] Ch. Labreuche and M. Grabisch. Bi-cooperative games and their importance and interaction indices. In 14th Mini-EURO Conference on Human Centered Processes (HCP'2003), pages 287-291, Luxembourg, May 2003.

[14] G. Owen. Game Theory. Academic Press, 3d edition, 1995.

[15] M. Sugeno. Theory of fuzzy integrals and its applications. PhD thesis, Tokyo Institute of Technology, 1974. 